\title{
Vision 2030 for the optimal approach to cancer research and care in Europe: A mission or a network of networks?
} \\ $\mathrm{TJ}$ thimom}

Tumori Journal

$1-6$

(c) Fondazione IRCCS-Istituto Nazionale dei Tumori

Reprints and permissions: sagepub.co.uk/journalsPermissions.nav DOI: $10.1177 / 0300891618783655$ journals.sagepub.com/home/tmj (S)AGE

\author{
Thierry Philip', Geneviève Almouzni' ${ }^{2}$ and Philip Poortmans ${ }^{3}$
}

\begin{abstract}
Introduction: A mission-oriented approach to cancer care in Europe was proposed by Julio Celis and Dainius Pavalkis in 2017. The major proposed objective is to achieve long-term survival of 3 out of 4 cancer patients by 2030.

Background: The authors are president or president-elect of Organization of European Cancer Institutes (OECI) EU Life or European Cancer Community Organization (ECCO).

Results: The goal is laudable and ambitious. However, it can only be successful if it is coordinated with active involvement of all stakeholders and interacts with an already well-organized, recognized, and certified European Accreditation and Designation quality approach to identify and select the potential candidates to participate in such a virtual network.

Conclusion: The building of a virtual European Cancer Institute based on coordinated networks should refer to unquestioned criteria using a solid and proven methodology operated by an independent arbitration body. The cancer community must share a fundamental responsibility to act collectively and in today's era of the World Wide Web, it is time to think out of the box and consider the possibility of establishing networks of networks.
\end{abstract}

\section{Keywords}

European cancer mission, network of networks

Date received: 3 May 2018; revised: 20 May 20I8; accepted: 29 May 2018

\section{Introduction}

A mission-oriented approach to cancer care in Europe was proposed by Julio Celis and Dainius Pavalkis in 2017. ${ }^{1}$ The major proposed objective is to achieve long-term survival of 3 out of 4 cancer patients by 2030 .

This goal is laudable and ambitious. Given the importance of the task, it may only be reached if the overall cancer community will be able to apply better the existing instruments to provide high-quality cancer care, offering to a higher percentage of cancer patients the possibility to benefit from the best available treatments. It should also be complemented by developing a capacity of early intervention to prevent the disease before it manifests. Therefore, if we want to reach the survival goal and prevent a disastrous disease outbreak in the future, important issues should first be debated at the European level:

- How do we facilitate interactions among and improve quality within the highest-volume cancer centers?
- How do we support the access to innovation and the growth of centers that have not yet reached a level enabling them to offer the best available treatments for a wide range of patients with cancer?

The cancer community must share a fundamental responsibility to act collectively, and in today's era of the World Wide Web, it is time to think outside the box and consider the possibility of establishing networks of networks.

\footnotetext{
Institut Curie, Paris, France

2Institut Curie Research Center, PSL University, CNRS, UMR3664, Sorbonne Universités, UPMC Paris, France

3Marie Curie Professor Paris Science \& Lettres, Institut Curie, Paris, France
}

Corresponding author:

Thierry Philip, Institut Curie, 73 rue Claude, Bernard, Paris 75005,

France.

Email: thierry.philip@curie.fr 


\section{The Challenge}

A landmark resolution on cancer prevention and control was adopted by member states at the World Health Assembly 2017, noting that "risk reduction has the potential to prevent around half of cancers" and urging "to promote cancer research to improve the evidence base for cancer prevention and control."

Cancer prevention is the most important challenge. ${ }^{2}$ Subsequently, screening and early diagnosis of cancer greatly increase the chance for successful treatment. Screening programs should be undertaken only when their effectiveness has been demonstrated, ${ }^{3}$ when resources are sufficient to cover the target group, and when facilities exist for confirming diagnosis and proceeding to treatment and follow-up. Based on the existing evidence, screening by population programs can be advocated only for breast cancer, cervical cancer, and colon cancer. ${ }^{4}$ More recently, targeted screening programs have been identified for well-specified populations at risk. Moreover, recognizing possible warning signs of cancer and taking prompt action lead to early diagnosis and in such a case surgery with or without radiation therapy is often sufficient to cure a majority of patients.

Early diagnosis and screening are the second major challenge to achieve long-term survival in Europe for patients with cancer.

Recent progress in the field of immunotherapy showed that we need to better understand the underlying mechanisms before using this new avenue of treatment for a broader group of patients. ${ }^{5}$ Fundamental research is the major way forward to structurally improve cancer survival in a non empirical way. The scientific outcome of fundamental research had helped us develop each individual tumor's specific genomic and molecular portrait as one of the ways towards precision medicine. ${ }^{6}$ Resolving spatial cellular heterogeneity, capturing cellular change in time, and establishing computational frameworks as parts of our understanding of the cause and biology of malignant diseases will further enhance our knowledge and thereby be a major step in the fight against cancer. ${ }^{7}$

Fundamental research is a third (but one of the most important in a long-term vision) contribution to approach the 2030 challenge of cancer in Europe.
Translational research is not only to work with industry in early clinical trials. It also includes the proof of concept that a laboratory discovery may be translated to applications in clinical practice, for which animal models remain of utmost importance.

Translational research should be hypothesis-driven and should support bench to beds studies. Early phase 0 and I studies are an important chapter but phase II and III and also phase IV studies in real life are part of this important challenge. ${ }^{8}$

Translational research, to transfer research discoveries from bench to bed and vice versa, is a fourth major challenge to achieve the targeted long-term survival of 3 out of 4 cancer patients by 2030 . Furthermore, reverse translation is equally important, since feeding back the most fundamental research based on data arising from studies with the patients is a necessary final means to the most effective iterative process to progress at all levels without working on each aspect separately.

\section{A Network of Networks: A Forward- looking Approach to Define a Mission- oriented Approach to a Virtual pan- European e-Cancer Institute Project}

The ongoing proposal concerning a Virtual European Cancer Institute has the potential to be promising. ${ }^{1}$ However, this can only be successful if it is coordinated with active involvement of all stakeholders and interacts with an already well-organized, recognized, and certified European Accreditation and Designation quality approach to identify and select the potential candidates to participate in such a virtual network.

A possibility, as briefly described below, is to set up coordination between currently existing networks that have the potential to evolve over time.

1. The main challenge is at the level of primary prevention, which could reduce the number of cancers by $30 \%$ to $40 \%$. Virtual networks already exist, mainly around Cancer Prevention Europe (Table 1), the European Network of Cancer Registries, the European Cancer Leagues, and a certain number of

Table I. Cancer Prevention Europe.

CPE has initially been established as a core group of 8 member institutes (Cancer Research UK, London; Danish Cancer Society, Copenhagen, Denmark; European Institute of Oncology, Milan, Italy; German Cancer Research Centre, Heidelberg, Germany; Imperial College London, UK; Karolinska Institute, Stockholm, Sweden; UK Therapeutic Cancer Prevention Network, Leicester; World Cancer Research Fund International, London, UK/Wereld Kanker Onderzoek Fonds, Amsterdam, the Netherlands), each committed to make an annual financial contribution within a formal Consortium Agreement together with a secretariat hosted by the International Agency for Research on Cancer, Lyon, France. The consortium funding will be used to appoint a senior Coordinating Scientific Officer with administrative support and an operating budget. This commitment will allow a focal point to coordinate development of the CPE priority actions within a 5-year Strategic Plan, including definition of the possible instruments to provide sustainability, the key partnerships, and relevant stakeholders. The CPE Consortium also includes the Maria SklodowskaCurie Institute-Oncology Centre, Warsaw, Poland, as an associate member. 


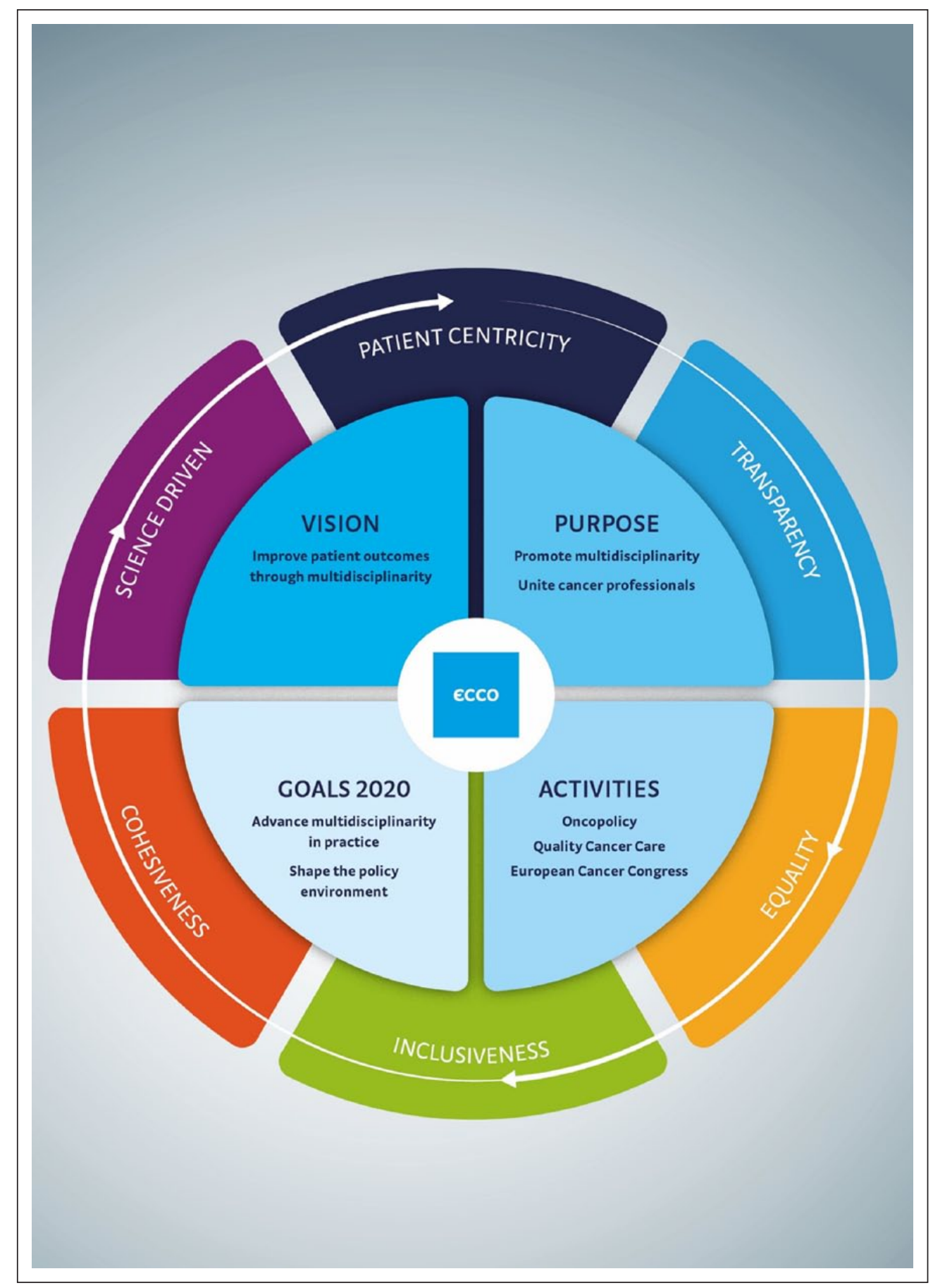

Figure I.

other groups. It should be straightforward to create this first network, perhaps in collaboration with international organizations such as International Agency for Research on Cancer-World Health Organization and International Union Against Cancer. $^{1}$

2. The European Cancer Organization (ECCO) (Figure 1), which brings together both cancer special- ists and patients, is entirely legitimate to establish a second network focused on the issues related to cancer care from screening over diagnosis, bridging hospital care with primary care levels (eccoorg.eu).

3. The third level is fundamental research to nurture our knowledge. The EU-LIFE alliance (Figure 2), for instance, represents an interesting model in 


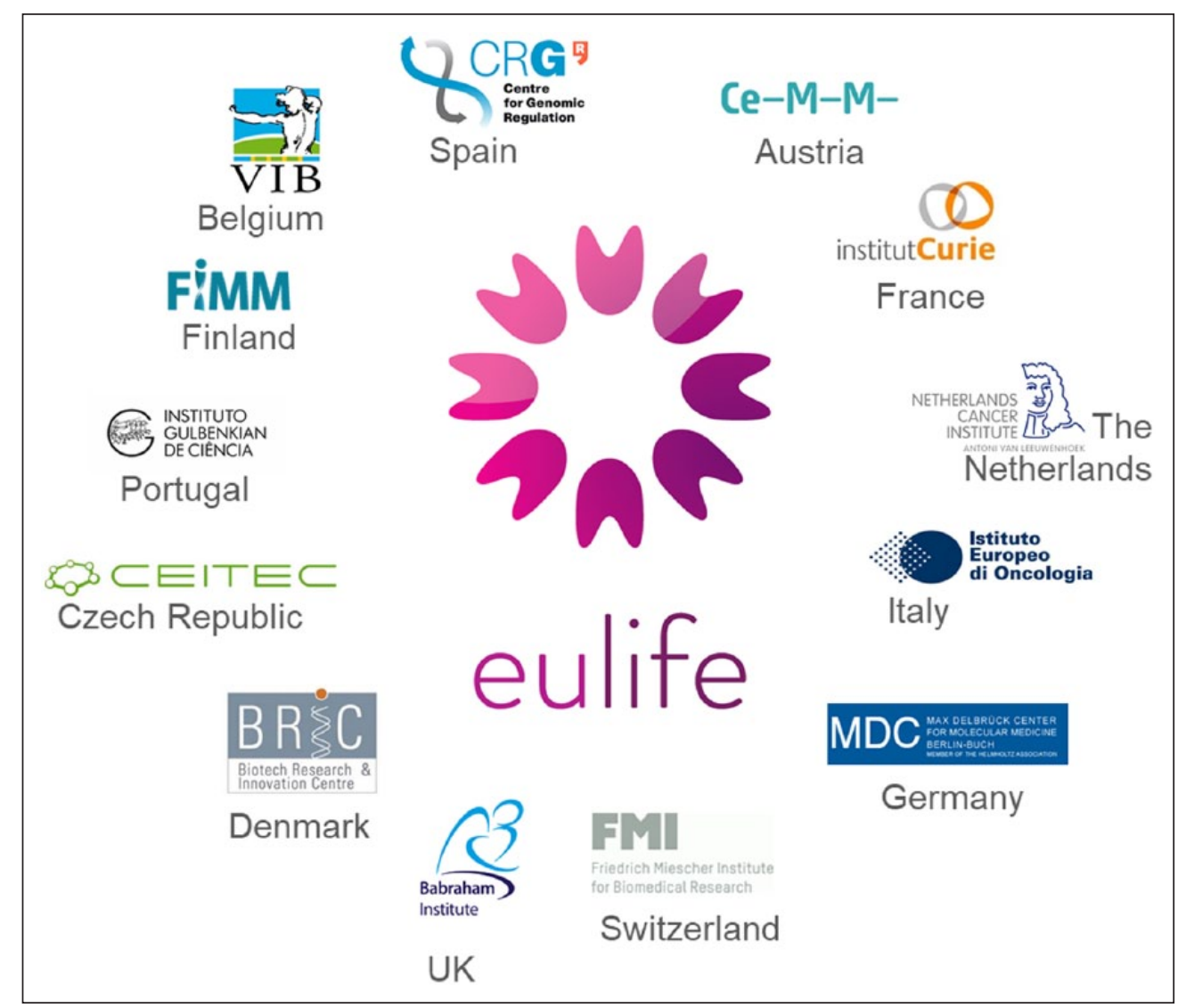

Figure 2.

Table 2. Cancer Core Europe.

Cancer Core Europe is a patient-centered infrastructure that aims at addressing the cancer research-cancer care continuum in partnership with major European cancer centers (Gustave Roussy Cancer Campus Grand Paris, the Cambridge Cancer Centre, the Karolinska Institutet, the Netherlands Cancer Institute, the Vall d'Hebron Institute of Oncology, and the German Cancer Research Centre with its CCC, the National Centre for Tumour Diseases and the Fondazione IRCCS Istituto Nazionale dei Tumori, Milano.). Cancer Core Europe was established following a bottom-up initiative led by A. Eggermont and O. Wiestler, triggered by the fact that it was not possible within the Eurocan Platform Network of Excellence project to find appropriate funding instruments that could be implemented within a reasonably short period of time. Being also a legal entity, Cancer Core Europe may serve as a hub and an engine to coordinate and optimize joint translational cancer research efforts across Europe. "Cancer Core Europe was exclusive at the start to become inclusive in the long term as a strong pillar was necessary to build and ensure the long-term success of the initiative."|

the spirit of an open approach. Starting with 13 of among the best European basic research centers, it aims to allow new members to join, based on specific inclusion criteria in a progressive manner and to foster links with other groups of institutions. These institutions already carry out research on cancer, have led several large European projects, and are actively engaged in future actions, among which is the preparation of a FET Flagship (lifetime-fetflagship.eu/).

4. Translational research represents one of the key objectives of both EU-LIFE and Cancer Core Europe $^{1}$ (Table 2). Together they could contribute to a fourth level on cancer translational research, including clinical research as well. However, new drug development is not sufficient to solve the issue of the fight against cancer by itself.

5. Cancer care and outcome research might be a field at the limits of European prerogatives. However, the topic of centralization of real-life data of patients treated for cancer is of great interest in terms of outcomes. A specific European network on this topic would be the last missing piece of the puzzle in order to create a Virtual European Cancer Institute. Given its links with the existing national networks, Organization of European Cancer Institutes (OECI) (Table 3) in relation with the Cancer Core initiative ${ }^{1}$ is in a 
Table 3. OECI.

$\mathrm{OECl}$ is a nongovernmental, nonprofit organization founded in Vienna in 1979 and remodeled in 2005 into OECI-EEIG, a European Economic Interest Grouping, headquartered in Brussels. As EEIG, based on the Council Regulation (EEC) No 2 I $37 / 85$ of 25 July $1985, \mathrm{OECl}$ is the only one, among all existing cancer organizations, having a European legal personality and the largest European EEIG as number of members and should be one of the key pillars of the network.

$\mathrm{OECl}$ gathers 90 member institutes, including some of the most prominent European Comprehensive Cancer Centres, Comprehensive Cancer Centres within universities, as well as other centers that are less involved in research and are simply designated as Cancer Centers.

$\mathrm{OECl}$ aims to create a critical mass of expertise and competences contributing to the production and dissemination of knowledge, so as to reduce fragmentation and increase competitiveness. These goals are being achieved by promoting and strengthening the concept of comprehensiveness and supporting quality in both cancer research and care, including a well-structured internal organization of these cancer centers. The final goal that should be obtained is to accelerate the production and implementation of personalized care approaches to ensure better treatments to all cancer patients, to produce more comprehensive care, and to improve patient quality of life through evidence-based medicine.

The Accreditation and Designation Programme is the $\mathrm{OECl}$ core business and aims to improve quality in cancer care and research, including the capacity to promote innovation. The OECI quality approach is ISQua certified as the only existing European process (with the German system) specifically designed to monitor the quality for cancer centers, while providing a significant impact on the organization of cancer care.

particularly strong position to contribute to the development of novel real-life approaches, raising the question of the price of innovative treatments.

In addition to university hospitals and cancer centers/ institutes, many general hospitals and private clinics are treating cancer without an established link with cancer research. As our efforts are patient-centered, we have to think about how these peripheral actors may interact with CCCs at a regional level and find their place as part of a network.

All the above levels are needed and should positively interact and collaborate owing to a solid though flexible and creative coordinating network. The head (the virtual European Cancer Institute) should be composed of a team of 20 to a maximum of 40 officers acting as conductors and facilitating the creativity and expression of the individual networks with a bottom-up approach representing the overall community.

\section{Selecting members of the networks: A major issue}

As far as translational research and clinical research is concerned, we need to select the CCCs in a first phase (cancer center or cancer centers within a university hospital) and focus on their capacity to join a network for translational research.

This is one of the issues OECI/Cancer Core Europe and several European Joint Actions are currently addressing. Indeed, the European Networks on Rare Cancers (rare solid cancers of adults, pediatric tumors, and rare hematologic malignancies) have proved to play a major role to ensure the same high level of quality of care for all patients with these rare malignancies.
The whole cancer community, together with the representatives of our patients, effectively linked to all domains of research, should communicate effectively in order to discuss and design the way Europe should act to guarantee a sustainable future for cancer research and care.

To achieve an open, multidisciplinary, democratic, and transparent approach including all the necessary talents, we have an existing model that fulfils the requirements: a model of integration and combination that translates differently depending on contexts, which may differ in terms of funding schemes and organization from one country to another, or organizational environments; for example, a cancer center compared to a university hospital. These models should not be exclusive, but on the contrary they should be united and complementary. Among the European cancer centers, members of OECI, currently 20 , are defined by OECI as CCCs within a university or with a link with the university. Designation as a CCC by OECI may be the first step to become part of a network for translational research.

In due course, therefore, other European CCCs are expected to join a putative mission based on their capacity to demonstrate scientific excellence. (EU-Life in particular will bring early translational research tools to such a network.) Cancer Core Europe in collaboration with EACS has developed quality criteria and methodologies for the designation of CCCs of excellence, defining in particular the level of integrative research to develop clinical applications. ${ }^{1}$ The method was already tested in practice during autumn 2014 and has been in use since the beginning of 2017.

Thereby, the designation of excellence for translational research may be a second step to select the members of the translational network. Centers in EU-Life are also players in the translational research field and connection among OECI, Cancer Core Europe and EU-Life may improve the process. 


\section{Conclusion}

The building of a virtual European Cancer Institute based on coordinated networks should refer to unquestioned criteria using a solid and proven methodology operated by an independent arbitration body. Quality and meritocratic criteria are the foundation on which to build such an initiative.

National networks already exist in many of the European countries. They must be involved in the process. This can only be achieved through the support of the concerned ministries and the needed cofounding to a project that cannot exclude risking the reflection by the main political actors of this historic challenge for the members' state.

Our organizations OECI, EU-Life, and ECCO are enthusiastic to be part of such a vision to build a real network including all European citizens to increase duration and quality of life everywhere in our community.

\section{Declaration of Conflicting Interest}

The authors declare that there is no conflict of interest.

\section{Funding}

This research received no specific grant from any funding agency in the public, commercial, or not-for-profit sectors.

\section{References}

1. Celis JE and Pavalkis D. A mission-oriented approach to cancer in Europe: a joint mission/vision 2030. Molecul Oncol 2017; 11: 1661-1672.

2. Forman D, Bauld L, Bonanni B, et al. Time for a European initiative to prevent cancer. A manifest for cancer prevention Europe. (Submitted 2018, personal communication.).

3. De Franch JJ, Barclay C and Sheridan S. The psychological harms of screening: the evidence we have versus the evidence we need. J Gen Intern Med (Epub 2014).

4. Esserman LJ, Thompson IM and Reid B. Addressing overdiagnosis and over treatment in cancer: a prescription for change. Lancet Oncol 2014; 15: 234-242.

5. Alatrash G, Jakher H, Stafford D and Mittendorf E. Cancer immunotherapies their safety and toxicity. Expert Opin Drug Saf 2013; 14: 631-645.

6. Byrne AT, Alférez DG, Amant F, et al. Interrogating open issues in cancer precision medicine with patient-derived xenografts. Nat Rev Cancer 2017; 4: 254-268.

7. Jaffee E, Van Dang C, Alexander B, et al. Future cancer research priorities in the USA: a Lancet Oncology commission. Lancet Oncol 2017; 18: 653-706.

8. Bentires-Alj M, Rajan A, van Harten W, et al. Stimulating translational research: several European life science institutions put their heads together. Trends Mol Med 2015; 21: 525-527. 